\title{
Optimal design of power frames for special purpose vehicles' cockpits with regard to their eigenfrequencies and shock resistance
}

\author{
Aleksandr Leontev ${ }^{1, *}$ Mikhail Aleshin ${ }^{1, *}$ Oleg Klyavin ${ }^{1, *}$ Alexey Borovkov ${ }^{1, *}$ \\ ${ }^{1}$ ST Petersburg State Polytechnical University, 195220 St.-Petersburg Gzhatskaya str. 21/ 2, Russia
}

\begin{abstract}
The paper is focused on development of a methodology for designing special purpose vehicles' cockpits minimizing vibration and impacts in the workspace. Finite elements method is utilized as the main tool in assessment of the structural design. The method also forms a foundation for further topology optimization, which allows for obtaining lightweight adaptable to production design fulfilling both vibration and impact requests, standards and regulations. The method has been used in designing experimental cockpit for a prospective tractor. Thus it is shown that the methodology being presented in the article can be successfully implemented in design of special purpose vehicles.
\end{abstract}

\section{Introduction}

Nowadays there is a trend of increasing interest to the problems of acoustics and vibration during the vehicle design. These are the factors, which a person directly interacts with. The rising concern on the topic is dictated by many factors:

- Desire to increase consumer satisfaction and, as a result, the vehicle competitiveness;

- Increase the computing power of modern computers, which allows solving large-scale problems, which were too consuming in terms of computation before.

\section{Problem description}

Tractor production is not an exception and the area has a rising interest to the NVH problems. The machine operator spends $80 \%$ of the working time in the cabin and, therefore, they are subjected to a constant affliction of vibrations and noise. As a result, when buying new equipment, the customers of the tractor pay special attention to the vibro-acoustic comfort of the cabin. Therefore, manufacturers of special equipment have to take into account the wishes of consumers and strive to reduce the noise and vibration.

Another important requirement for the cabin of special machinery is the presence of a protective structure. Satisfaction of this requirement ensures the safety of the tractor operator in the case of dangerous and emergency situations: tractor roll-over and heavy objects drop on the roof. These requirements are regulated by international standards, technical regulations of the Eurasian Customs Union, GOST standards. Its fulfillment is mandatory for all manufacturers of special vehicles.
Therefore, the developers of new technology face the need to meet the mutually exclusive requirements imposed on the design in attempts to increase vibroacoustic comfort, as well as passive safety. For example, the tractor cabin must be rigid so the lowest natural frequency would be high enough, and, at the same time, it must be sufficiently compliant to maximize impact energy absorption during the roll-over.

In accordance with this, it becomes necessary to develop an instrument that will provide an opportunity of obtaining the optimal solution that will satisfy mutually exclusive requirements for vibro-acoustics and passive safety in an automatic mode.

\section{Goals and objectives}

Within the framework of the project funded by the Ministry of Education of the Russian Federation, we set the goal to develop such a tool while creating a next-gen tractor with improved design, visibility, ergonomics, vibro-acoustic and climatic comfort.

At the first stages of the project, a digital model of the original tractor design was created in order to determine the layout constraints on the design. Next, an updated style surface and the power frame of the cabin was developed were designed. The current focus is on fine-tuning of the cabin design and layout (Fig. 1).

\footnotetext{
*Corresponding author: Leontev@compmechlab.ru; aleshin@compmechlab.com; klyavin@compmechlab.com; Borovkov@compmechlab.com
} 


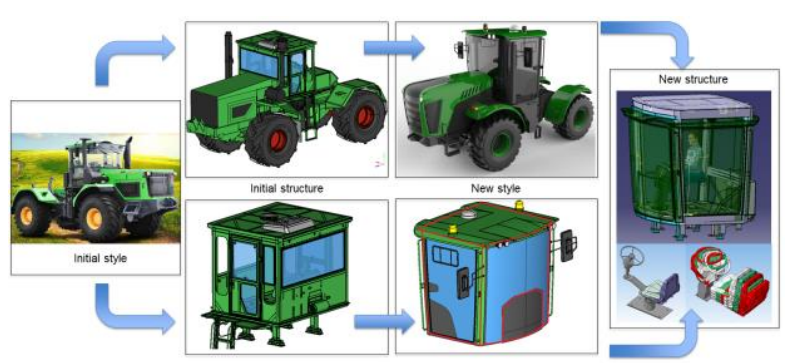

Fig. 1. The process of the development of the new cabin design.

To achieve this goal, it was necessary to solve the following tasks:

- Considering the classical approaches to the design of special equipment cabins;

- Set up the goals and limitations for optimization by cascading targets and constraints from the subjective wishes of the customer to the technical requirements for the nodes and components;

- Development of an algorithm for multidisciplinary cross-platform structural optimization;

- Carry out a multi-disciplinary cross-platform optimization of the power frame of the tractor cab.

\section{The classical approach to the design process}

The classical approach to design is based on the results of topology optimization [1]. First of all, the analysis of layout and style surface is carried out and the scope of the design is determined, that is, the volume in which the structural elements of the structure can potentially be located (Fig. 2).

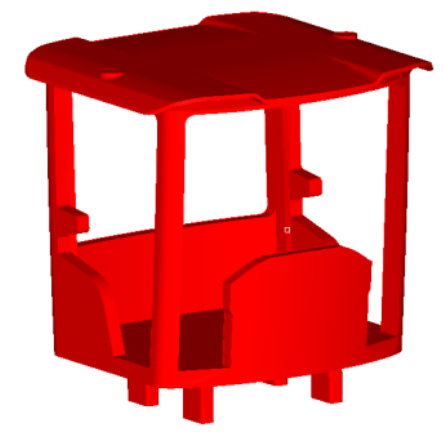

Fig. 2. Design volume.

After this, topology optimization is carried out. Its results determine the main force paths of the power frame (Fig. 3).
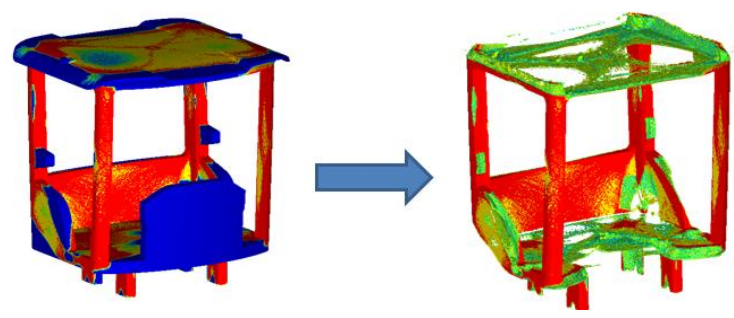

Fig. 3. Results of topology optimization.
Then a beam frame model is constructed and parametric optimization of the beam cross-sections is performed (Fig. 4).

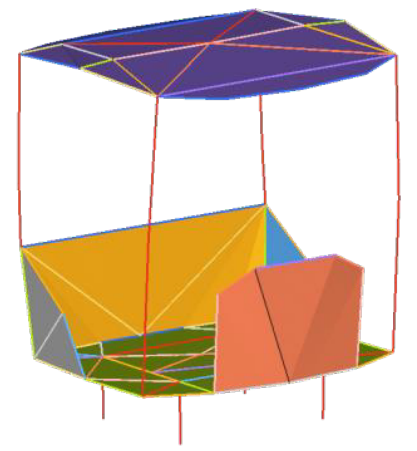

Fig. 4. Beam model of the tractor cab.

As a result, a section map is constructed. Taking is as a basis a mathematical model of the prototype of the power frame is developed. Further, a multi-variant modification of the structure is performed separately for vibro-acoustic comfort and for passive safety. Often, those design solutions that have a positive effect on vibro-acoustic comfort have a negative effect on passive safety and vice versa. Thus, the process of creating a design that satisfies the set goals is multi-iterative and takes a long time.

All in all, the classical approach requires a large number of highly qualified stuff, it takes a lot of time and does not give the most effective result.

\section{Algorithm of multidisciplinary cross- platform optimization}

The process of obtaining the optimal solution using multidisciplinary cross-platform optimization for the most part resembles the process of the classical approach, but it has drastic differences at the stage of the design finalization. Instead of manual refinement of the design, optimization is used at each iteration to analyze both vibro-acoustic parameters, such as the global natural frequencies of the design and the parameters of passive safety, such as the penetration of structural elements into the cabin inner space. In the end of each iteration the engineer determines whether the targets are met. If the requirements are not fulfilled, the optimization variables are altered and the next iteration is performed (Figure 5).

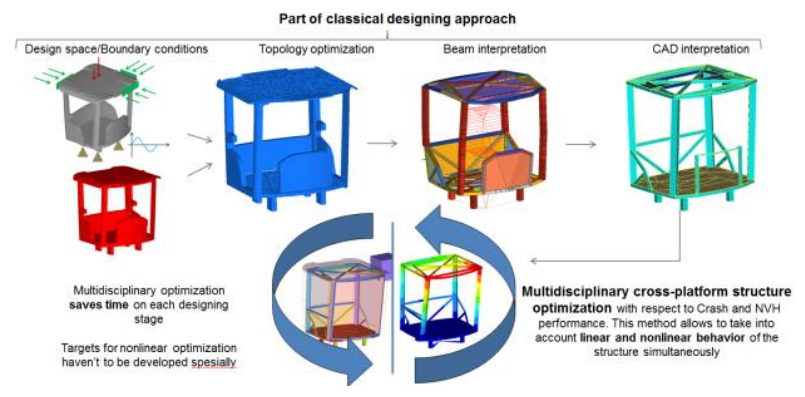

Fig. 5. The process of multidisciplinary cross-platform optimization. 
Optimization is considered converged if all targets are met.

Despite its advantages, multidisciplinary crossplatform optimization has a number of limitations:

- The topology of the design does not change during optimization;

- The shape change in the optimization process is limited

\section{Variables, boundary conditions and constraints for optimization}

Analysis of the NVH main requirements and their further cascading showed that one of the main parameters are global natural frequencies of the cabin on its own suspension. Requirements for these indicators are directly related to the excitation frequencies from the power unit. Own frequencies of the cabin should square root of two larger than the frequency of the installed engine idle.

Target values for natural frequencies were calculated based on the frequency of the dominant motor harmonics for the internal combustion engine according to the formula (1). The project took into account the characteristics of the engines YaMZ 238 and TMZ 84;

For V-type engine:

$$
f=\frac{0.5 \cdot n i}{60 \cdot \tau},
$$

$\mathrm{i}$ is the number of cylinders, $\tau=1$ for a two-stroke engine, $\tau=2$ for a four-stroke engine.

The selected target values are guaranteed to ensure compliance with the requirements for vibro-acoustic comfort:

- target values of the frequencies of the torsional vibrations of the assembled tractor cab roof relative to the floor and supports around the $\mathrm{Y}$ axis were set in the range of more than $25 \mathrm{~Hz}$ and more than $30 \mathrm{~Hz}$, respectively;

- frequency of natural oscillations of the assembled tractor cab in the longitudinal direction - in the range from 11 to $18 \mathrm{~Hz}$ and more than $25 \mathrm{~Hz}$;

- natural frequencies of the assembled tractor cab in the transverse direction - in the range from 16 to $18 \mathrm{~Hz}$ and more than $25 \mathrm{~Hz}$.

Analysis of the basic requirements for passive safety and their cascading showed that the main indicators are the maximum displacement of the structural elements of the cabin under the impact of shock loads.

Thus, the optimization goal is to reduce the mass, while limiting the lowest global natural frequency of the cabin's power frame, according to the maximum penetration of the pendulum with lateral and rear impacts.

The design optimization variables are the thicknesses of all the beams (Figure 6) that make up the power frame of the cabin. In addition to this, the shape of the cabin is a design variable as well. Variables are width, length, height of the cabin, the angle of inclination of the windshield, as well as the angle of inclination of the side walls of the cabin.

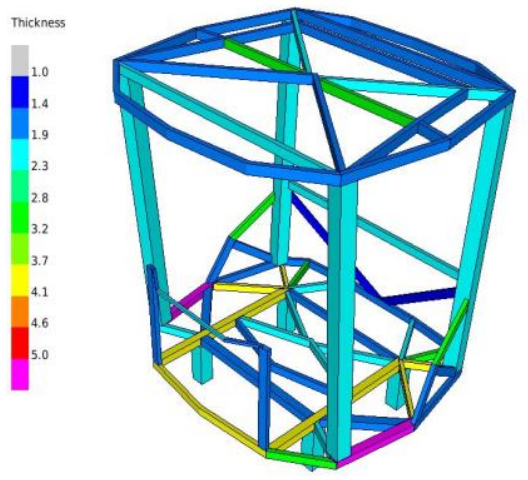

Fig. 6. Parts whose thicknesses are alerting during optimization.

\section{Results of multidisciplinary cross- platform optimization}

During the optimization process 57 iterations were carried out. It took 136 hours. As a result, the design of the tractor cab which satisfies all the requirements was obtained (Fig. 7,8):

- The first global natural frequency increased from 19.8 $\mathrm{Hz}$ to $25.2 \mathrm{~Hz}$;

- The second global natural frequency increased from $22.6 \mathrm{~Hz}$ to $28.5 \mathrm{~Hz}$;

- The third global natural frequency increased from 26.3 $\mathrm{Hz}$ to $32 \mathrm{~Hz}$;

- Penetration of the pendulum with a lateral impact decreased from $261 \mathrm{~mm}$ to $215 \mathrm{~mm}$;

- Penetration of the pendulum with a rear impact dropped from $204 \mathrm{~mm}$ to $194 \mathrm{~mm}$;

- The weight of the power frame decreased from 709.6 $\mathrm{kg}$ to $656.2 \mathrm{~kg}$.

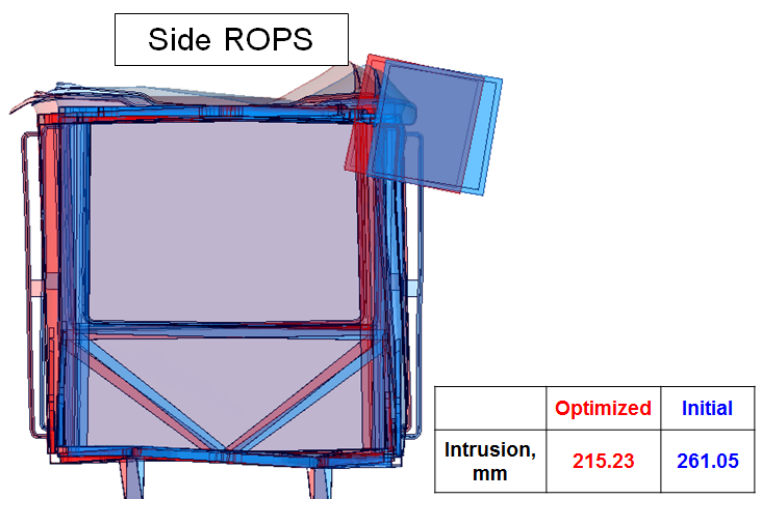

Fig. 7. Comparison of optimized and non-optimized side impact structures. 


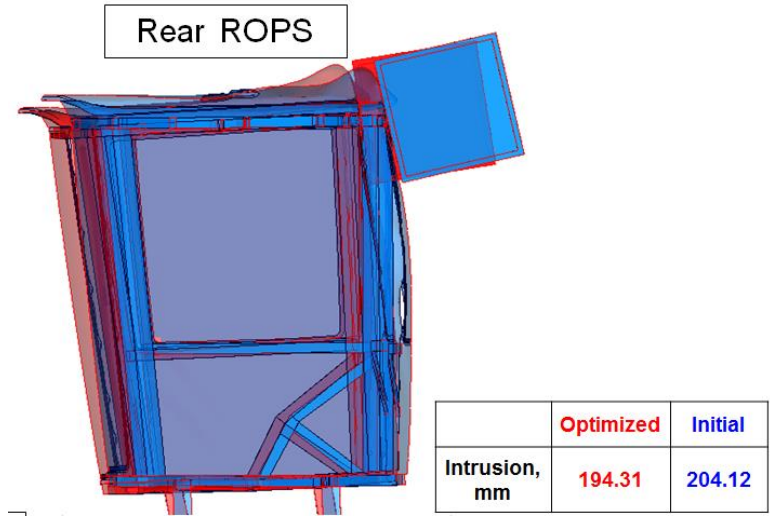

Fig. 8. Comparison of optimized and non-optimized designs in the case of the rear impact.

\section{Specifications of the computing server}

Optimization was carried out on the HPC of SPBPU, which is on the third place in Russia in terms of performance and is the most powerful computer in Russia, which is used to solve applied problems of various industries of the Russian Federation. The performance of the HPC is 1.3 Pflops (Figure 10).

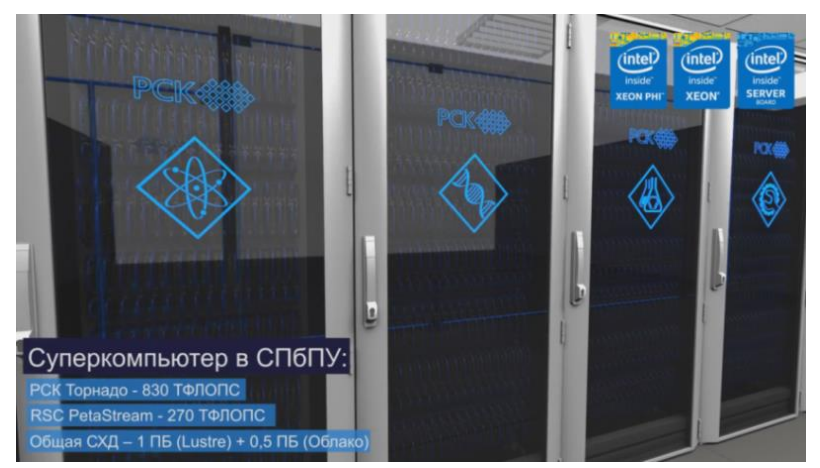

Fig. 9. The used HPC.

\section{Main conclusions}

The outcome of the performed actions is the tractor cab structure, which:

- Has a reduced mass

- Satisfies the vibro-acoustics rquirements

- Satisfies Passive Safety Requirements

Thus, it can be concluded that the approach of multidisciplinary cross-platform optimization for the designing procedure of power frames of special vehicles leads to reduction of production costs (be the means of material expenses reduction), design time reduction, and it also allows to obtain an optimal result while taking both requirements for vibro-acoustics and passive safety into account.

The work is carried out with financial support of the Ministry of Education and Science of the Russian Federation within the framework of the Federal Program "Research and Development in Priority Areas for the Development of the Russian Science and Technology Complex for 2014-2020",
Activity 1.3., Agreement on the Grant No. 14.578.21.0206 of 03.10.2016, the unique identifier of the program of research and experimental works: RFMEFI57816X0206..

\section{References}

1. Bendsoe M.P., Sigmund O Theory, Methods and Applications. 2nd ed. Berlin: Springer-Verlag, 2004. 370 p. 